山्山FFRANÇAISE

$\supset \mathrm{DE}$

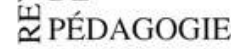

\section{Revue française de pédagogie}

Recherches en éducation

$185 \mid 2013$

L'enseignement de la musique, entre institution scolaire et conservatoires

\title{
L'enseignement de la musique dans un contexte inhabituel : le cas d'un atelier musical pour parents et enfants de 0-3 ans
}

The teaching of music in an unusual context: the case of a music workshop for parents and 0-3 year old children

\section{Géraldine Bois}

\section{(2) OpenEdition}

Journals

Édition électronique

URL : http://journals.openedition.org/rfp/4340

DOI : 10.4000/rfp.4340

ISSN : 2105-2913

Éditeur

ENS Éditions

Édition imprimée

Date de publication : 31 décembre 2013

Pagination : 59-68

ISBN : 978-2-84788-523-1

ISSN : 0556-7807

\section{Référence électronique}

Géraldine Bois, «L'enseignement de la musique dans un contexte inhabituel : le cas d'un atelier musical pour parents et enfants de 0-3 ans », Revue française de pédagogie [En ligne], 185 | 2013, mis en ligne le 31 décembre 2016, consulté le 19 avril 2019. URL : http://journals.openedition.org/rfp/4340 ; DOI : 10.4000/rfp.4340 


\section{L'enseignement de la musique dans un contexte inhabituel : le cas d'un atelier musical pour parents et enfants de 0-3 ans}

L'article vise à déterminer la place donnée à la musique et la forme d'enseignement à l'œuvre dans un contexte inhabituel : celui d'un atelier musical pour des enfants de moins de trois ans et leurs parents, organisé au sein d'un conservatoire en partenariat avec des services sociaux. Pour ce faire, il analyse non seulement les objectifs de cet atelier et les pratiques et conceptions pédagogiques du musicien intervenant qui l'anime, mais aussi les appropriations qu'en font les parents. En fonction de leurs caractéristiques sociales et des manières dont ils ont accédé à l'atelier, ceux-ci interprètent plus ou moins les séances dans un sens musical et scolaire. Leurs appropriations contribuent ainsi à définir ce qui, de cette activité, est transmis à leurs enfants.

Mots-clés (TESE) : école de musique, enfant, parents, partenaires sociaux, situation sociale.

Dans les conservatoires ${ }^{1}$, le premier cycle d'études musicales accueille les enfants à partir de sept ou huit ans. C'est donc à partir de cet âge que débutent la pratique instrumentale et/ou vocale et la formation musicale ${ }^{2}$ proprement dites, sanctionnées par des évaluations et des examens de fin de cycle. Avant cet âge, les établissements peuvent proposer, à partir de cinq ans, des années d'"éveil musical» puis d'«initiation» ou de "découverte", afin de préparer les enfants aux études musicales et de les aider à choisir leur discipline et leur instrument. Si les activités musicales conçues pour des enfants plus petits sont encouragées (Ministère de la Culture et de la Communication, 2008, p.4), elles sont plus rares et s'organisent généralement hors-les-murs, dans des structures en charge de la petite enfance (crèches et écoles maternelles). Dans tous les cas, ces activités organisent un face-à-face entre un ou des enfants $^{3}$ et un enseignant du conservatoire (avec la présence plus ou moins active d'autres professionnels - de la petite enfance en l'occurrence - dans le dernier cas).

Ainsi l'atelier musical sur lequel porte cet article est-il original pour plusieurs raisons : il est destiné à de très jeunes enfants non scolarisés (entre zéro et trois ans) ${ }^{4}$, il est organisé en partenariat avec d'autres structures mais dans les locaux d'un conservatoire, et il se déroule en présence des parents qui participent activement aux séances aux côtés de leurs enfants. Les professionnelles des structures partenaires - essentiellement le Service petite enfance et le Service social de la ville, et secondairement la Protection maternelle infantile - ne sont pas présentes lors des séances. Leur rôle consiste à orienter des familles vers l'activité et à gérer l'inscription de toutes les familles (même si une famille vient s'inscrire «spontanément», elle 
doit le faire auprès de ces services et non pas directement au conservatoire). Ces modalités d'inscription renforcent le caractère marginal de cet atelier musical en lui donnant une place un peu à part au sein du conservatoire. En effet, si l'enseignant qui anime les séances dispose bien d'une liste d'inscrits, ces derniers ne sont pas comptabilisés parmi les élèves de l'établissement.

L'objectif de cet article est d'interroger la place donnée à la musique et la forme de «l'enseignement »5 dispensé dans ce contexte inhabituel de transmission de la pratique musicale, en s'attachant d'une part aux objectifs de l'atelier et aux pratiques et conceptions pédagogiques de l'enseignant qui l'anime, et d'autre part aux appropriations parentales des séances. Comme toute socialisation secondaire, la transmission d'une pratique musicale en dehors du cadre familial dépend de ce qui se passe dans le contexte d'enseignement concerné mais aussi des expériences socialisatrices que les enfants ont vécues au sein de leur famille et qui orientent leur appréhension de cette pratique. Ce rôle de «filtre » joué par la socialisation primaire (Berger \& Luckmann, 1986) est sans doute d'autant plus fort que les agents de cette socialisation sont physiquement présents pour l'exercer. Autrement dit, en tant que participants actifs aux séances, les parents contribuent directement à la définition du contenu et de la forme de l'enseignement transmis à leurs enfants.

Dans la première partie de l'article, nous analyserons les objectifs de l'atelier et les pratiques et conceptions pédagogiques mises en œuvre en son sein, afin de déterminer la place donnée à la musique dans cette activité et la forme d'enseignement privilégiée. L'atelier s'avère relativement éloigné à la fois de la pratique musicale au sens strict (c'est-à-dire ne faire que de la musique dans un objectif musical) et de la forme scolaire (ce qui ne signifie pas, comme nous le verrons, qu'aucun mode de socialisation scolaire n'est à l'œuvre). La seconde partie sera consacrée à l'étude des appropriations parentales socialement différenciées de l'atelier, avec l'idée que le sens particulier que les parents lui donnent contribue à définir ce qui est transmis in fine à leurs enfants. Nous verrons ici que les parents appartenant aux milieux sociaux favorisés ont davantage tendance à «musicaliser» et à «scolariser» les séances que les parents de milieux populaires.

\section{OBJECTIFS ET FORME D'ENSEIGNEMENT}

\section{Des objectifs sociaux plus que musicaux}

Du fait de l'implication des services sociaux dans sa genèse et du public visé, les objectifs de l'atelier étudié renvoient principalement au domaine du travail social. L'atelier est ainsi conçu comme un outil de soutien à la parentalité et d'insertion sociale des familles, en vue de prévenir - de manière la plus précoce possible - les conséquences négatives que des «problèmes» relationnels pourraient avoir sur le «bon» développement des enfants ${ }^{6}$. L'activité s'adresse en priorité aux milieux populaires, soit parce que les professionnelles de la petite enfance qui reçoivent des familles pour diverses raisons (information sur les modes de garde par exemple) perçoivent chez certaines d'entre elles une «carence » éducative ou un isolement à prendre en charge, soit parce que les assistantes du Service social sont de fait confrontées à un public «en difficultés » (celles que nous avons interrogées disent ainsi proposer l'activité à tous les parents qu'elles rencontrent dont l'enfant a l'âge requis) 7 . L'orientation vers l'atelier musical s'inscrit ainsi dans un contexte d'institutionnalisation de la «cause de l'enfant», dans lequel des familles de milieux populaires sont exposées à des normes éducatives propres aux milieux favorisés (Garcia, 2011).

II s'agit tout d'abord dans cet atelier de favoriser les liens parent-enfant, c'est-à-dire d'«offrir un temps de socialisation où parents et enfants partagent un moment de plaisir au travers d'une activité d'éveil, où le parent se retrouve premier éducateur de son enfant et où le lien affectif parent-enfant est renforcé »8. L'atelier est aussi conçu comme un support du lien social à une échelle plus large, favorisant les rencontres entre familles, au sein des séances mais aussi dans le quartier, par le biais notamment d'une «fête de la musique des tout-petits » organisée dans un square très fréquenté à proximité du conservatoire. On retrouve ces deux objectifs dans les propos de la responsable du Centre information petite enfance et des assistantes sociales, pour qui il s'agit avant tout de rompre l'isolement parental (en particulier maternel), de permettre aux parents de faire une parenthèse dans leurs problèmes, de «nourrir la relation parentenfant» en partageant une expérience «agréable» et "positive», de redonner confiance aux parents dans leurs fonctions éducatives, etc. L'enseignant du conservatoire en charge de l'atelier partage pleinement ces objectifs, d'autant plus que c'est lui qui est à l'origine du projet, élaboré dès le début en concertation avec sa «collègue " du Service petite enfance avec qui il continue de collaborer étroitement pour l'organiser chaque année9. 


\section{L'enquête}

Cet article est basé sur une enquête menée en 2012 et 2013 dans le cadre d'une recherche collective (Bois, Gardon, Giraud et al., 2014), financée par la Région Rhône-Alpes, consacrée à la démocratisation de l'enseignement artistique (musique, danse ou théâtre). Six actions de «démocratisation» menées par des conservatoires de la région ont été étudiées. L'atelier musical dont il est question dans cet article existe depuis plus de dix ans; il est gratuit et réservé aux habitants de la ville. Il fait partie des actions étudiées parce qu'il s'adresse en partie à des familles de milieux populaires orientées par les services partenaires. Le conservatoire municipal de musique ${ }^{10}$ dans lequel il a lieu est situé dans un quartier excentré d'une ville d'environ 32000 habitants, en Zone urbaine sensible. Les services partenaires occupent des locaux situés dans le même quartier, en face du conservatoire.

L'analyse de l'atelier mobilise trois types de matériaux : 1) des analyses de documents (document de présentation de l'atelier par les services de la ville, plaquettes de présentation des activités du conservatoire, projet d'établissement); 2) des observations de trois séances d'atelier de quarante-cinq minutes chacune, accueillant au maximum douze familles; 3 ) treize entretiens, dont cinq avec des représentants des structures engagées dans le projet (le directeur du conservatoire, l'enseignant en charge des séances, la responsable du Centre information petite enfance et deux assistantes sociales du Service social municipal), et huit avec des parents (six mères, un père et un couple) concernés par l'atelier, soit parce qu'ils y participent au moment de l'enquête (pour cinq d'entre eux), soit parce qu'ils y ont participé quelques années auparavant (pour trois d'entre eux).

Les huit familles ont des caractéristiques variées qui déterminent leurs appropriations de l'atelier. Du point de vue des propriétés sociales tout d'abord, quatre appartiennent aux classes populaires, trois aux classes moyennes et une aux classes supérieures. En outre, six ont été orientées vers l'atelier par l'un des services partenaires alors que les deux autres sont venues s'inscrire d'elles-mêmes (il s'agit de la famille de classe supérieure et d'une des familles de classe moyenne).

Toutes les familles rencontrées ont été contactées par l'intermédiaire de Rémi11, le musicien intervenant du conservatoire qui anime l'atelier. Celui-ci les a appelées pour leur demander leur accord pour un entretien avant de nous fournir leurs coordonnées. Les familles enquêtées sont donc à la fois suffisamment réceptives à l'atelier musical pour que Rémi les estime intéressées par une participation aux entretiens, et suffisamment en bons termes avec Rémi pour accepter sa proposition (de fait, grâce à son intermédiaire, l'accueil des familles a toujours été chaleureux). Par exemple, l'une des mères rencontrées dit avoir accepté de réaliser l'entretien parce qu'elle est "contente d'avoir eu l'occasion de faire cet atelier». Si toutes les familles qui continuent à se rendre aux séances une fois passées les premières y trouvent forcément un intérêt (de quelque nature qu'il soit), les familles enquêtées sont donc particulièrement bien disposées à leur égard. L'analyse permet de connaître les appropriations de l'atelier par ces familles-là. En d'autres termes, elle met au jour des résultats qui ne sont pas forcément représentatifs de toutes les familles qui participent à cette activité, et a fortiori de celles qui l'ont abandonnée.

Pour cet enseignant comme pour ses partenaires, l'activité musicale n'est pas pratiquée pour elle-même mais elle est davantage un outil. "Le support c'est la musique, mais [...] dans un objectif social, de construire du lien pour les familles", dit par exemple la responsable du Centre information petite enfance. Cet ordre de priorité apparaît clairement lorsqu'elle dit ne pas proposer l'atelier «musique» aux parents qui fréquentent déjà l'autre "atelier de parentalité" proposé par la ville ${ }^{12}$ parce que, selon elle, cela ferait "doublon». La spécificité musicale de l'activité est ici mise au second plan. Rémi pense lui aussi la musique pratiquée lors de son atelier comme une source de plaisir permettant avant tout de nourrir la relation parent-enfant, voire d'aider des parents «en difficultés " à accéder à leur rôle d'“éducateur premier » de leur enfant. De manière significative, lorsque sont abordées avec lui des questions de progression dans la pratique des participants aux ateliers, il ne mentionne que la progression au sens relationnel du terme, en soulignant que des parents qui avaient peu de relations avec leur enfant en début d'année «vont être dans le jeu avec [lui]» en fin d'année. De fait, parmi les propositions musicales de Rémi, plusieurs sont conçues pour susciter ces liens parent-enfant, tout en s'adaptant au très jeune âge des enfants. Ce sont par exemple des chansons qui incitent "à faire rebondir l'enfant sur les genoux, ou à le faire tomber par terre, ou qui se prêtent bien à danser ensemble". Lors des séances observées, nombreuses sont en effet les activités qui favorisent une complicité et un contact physique. Une chanson s'accompagne d'un jeu qui consiste pour l'enfant à mettre sa main dans la chaussette de sa mère, une autre incite parents et enfants à se chatouiller et, dans le cadre d'une comptine, Rémi passe de famille en famille avec un grand papier avec lequel il cache le visage du parent puis le dévoile en s'exclamant : "Cachée maman!», «trouvée!»13.

La place que Rémi donne à la musique dans son enseignement s'explique aussi en partie par son statut et par sa formation (Lebon, 2014). Selon lui, en effet, un musicien 
intervenant appréhende la musique comme un «moyen» et non pas comme «un but en soi». En mettant l'objectif musical au second plan, Rémi met aussi à distance la forme scolaire de transmission des savoirs, qui implique notamment de considérer une discipline pour elle-même, sans se préoccuper de son utilité (Vincent, 1994). II se définit d'ailleurs comme un «éducateur » plutôt que comme un «enseignant», et souligne très souvent pendant son entretien la distance qui le sépare des «professeurs» du conservatoire.

\section{Un éloignement relatif de la forme scolaire}

L'atelier animé par Rémi est loin d'être informel. II se caractérise en effet par un cadre spatio-temporel clair, par la présence d'un adulte qui oriente les séances, et par une série d'apprentissages au cours desquels la musique est utilisée comme un outil d'éducation des jeunes enfants. II s'agit de leur apprendre à attendre, à ne pas toucher, à ranger, à côtoyer d'autres enfants, etc. Par exemple, à chaque séance, Rémi utilise une chanson rituelle pour amener les enfants à venir ranger le matériel dans un grand sac. II pense en outre que l'atelier joue un rôle dans leur apprentissage des limites : «ça fait aussi partie des objectifs, le "non", la canalisation, le fait que y ait un temps pour ça mais il viendra plus tard. "C'est pas maintenant que tu as le droit de prendre l'instrument, mais je t'en prêterai un plus tard" ». L'atelier étudié participe ainsi d'un mouvement de «pédagogisation » des activités des jeunes enfants, caractérisé par une diffusion des normes scolaires dans les lieux chargés de les accueillir ou d'organiser leurs loisirs (Chamboredon \& Prévôt, 1973; Garnier, 2006, 2009; Luc, 1997). Ces normes sont partagées par les professionnelles des services sociaux qui orientent les familles, si bien que l'objectif - déjà souligné - de soutien éducatif des parents de milieux populaires prend aussi la forme d'une «pédagogisation» de leurs pratiques éducatives. L'enseignement dispensé au cours des séances d'atelier reste cependant relativement éloigné de la forme scolaire pour trois raisons principales, qui sont liées au très jeune âge des enfants (non scolarisés) mais aussi aux conceptions et pratiques pédagogiques de Rémi et à l'orientation du conservatoire où il exerce.

La première de ces raisons tient au faible degré d'autonomie de la musique telle qu'elle est pratiquée dans l'atelier. Là où la scolarisation d'une discipline suppose de la distinguer des autres, on a affaire au sein des séances à un mélange d'activités. Les propositions de Rémi relèvent bien du domaine musical et consistent en des chansons, des comptines, de l'expression corporelle et une utilisa- tion de petits instruments ou de petits objets qui émettent des sons (du papier froissé par exemple). Mais ces éléments musicaux sont mêlés à d'autres activités non spécifiquement musicales comme des empilements d'objets, des jeux de cache-cache, etc. Si la nature de ces activités est pensée comme adaptée aux enfants («C'est une chanson que je fais évoluer avec des objets qu'on empile les uns sur les autres, parce que y a le plaisir de l'enfant d'empiler et de détruire ce qu'on vient d'empiler, de construire et de recommencer », explique par exemple Rémi), c'est aussi le fait même de ne pas distinguer les différentes activités qui est mis en avant. La comparaison avec l'enseignement scolaire est ici explicite : «L'enfant est jeune, et comme il est jeune, il est dans la pluridisciplinarité, il vient pas là uniquement à se dire : "On fait que musique, et puis l'heure d'après on fait mathématiques, et puis après on fait français" ». Si la présence d'activités non strictement musicales n'annule pas la dimension musicale de l'atelier, elle peut en revanche contribuer à masquer cette dimension, comme nous le verrons au moment d'analyser les appropriations des séances.

C'est aussi la mise en œuvre par Rémi d'une conception de la pratique musicale comme un «plaisir» «sur l'instant » et non pas comme une discipline à travailler, et à travailler sur le long terme, qui éloigne son atelier de la forme scolaire. Il n'y a jamais lors des séances d'injonctions à «bien faire » ou à progresser d'un point de vue musical, qu'elles soient adressées aux enfants ou aux parents. De plus, si Rémi constate bien, à l'issue de l'atelier, quelques cas de pérennisation de la pratique musicale au sein de l'établissement, il ne les présente jamais comme un résultat souhaité. S'il est d'accord avec les professeurs du conservatoire qui lui disent que tout le monde n'est pas capable de faire de la musique à «un niveau élevé ", il estime que tout le monde est par contre capable «d'avoir une pratique musicale» (c'est-à-dire pas forcément à un haut niveau et pas forcément via une pratique instrumentale). Cette manière de considérer la pratique musicale n'est pas seulement liée au fait qu'il a affaire à des enfants qui n'ont pas l'âge de fréquenter le cursus d'études musicales ou au fait qu'il s'adresse en partie à des familles de milieux populaires pour qui la poursuite d'une activité musicale n'est pas perçue comme une priorité ${ }^{14}$. II la met en œuvre pour les enfants de tous âges et pour les adultes, en lien avec sa formation de musicien intervenant ${ }^{15}$, qui l'a amené à ne pas considérer la musique comme « un but à atteindre ou une discipline technique» mais à favoriser au contraire la «sensorialité » et le «plaisir» (qu'il oppose justement à la «technique», qu'elle soit instrumentale ou liée à la maîtrise du solfège). 
Enfin, au niveau du type d'autorité exercée, l'enseignement de Rémi est basé sur un degré faible de réglementation - explicite et implicite - des attitudes requises lors des séances et par un fort degré de personnalisation des relations enseignant-élèves. Rémi ne fait aucun commentaire lorsque des parents sont en retard ou sortent au milieu de la séance. De plus, il ne donne aucune information préalable sur ce qui va être fait ou sur ce qu'il faut faire pendant l'atelier, et il entre directement dans la pratique dès le début de la séance. Pendant la séance, les consignes explicites sont également absentes et, si les activités elles-mêmes peuvent fonctionner comme des consignes implicites, les attentes de Rémi sont faibles. Par exemple, il ne s'interrompt quasiment jamais lorsque les enfants font tout autre chose que l'activité qu'il est en train de leur proposer (courir dans la salle plutôt que chanter par exemple). En entretien, il critique l'attitude de certains parents qui veulent soit que leur enfant reste assis calmement à écouter, soit que leur enfant fasse les mêmes gestes que lui (taper dans ses mains quand il tape dans les siennes par exemple). Enfin, Rémi est loin d'incarner une autorité impersonnelle. II accueille systématiquement les familles dans le hall d'entrée du conservatoire, prodigue des bonjours individualisés aux parents et aux enfants, dit au revoir en embrassant les enfants qui le souhaitent et entretient avec les parents une relation de connivence, basée sur le partage d'expériences ordinaires et sur la plaisanterie : «Ah ouais, c'est pas simple pour nous les parents! ", dit-il par exemple à une mère dont l'enfant a beaucoup pleuré lors de la séance; "J'aime bien plaisanter avec les parents parce qu'on se voit chaque semaine, $[. .$.$] on a un vécu commun, quoi, [...] donc des$ fois j'aime bien utiliser l'humour comme moyen de lien, mais... comme je ferai avec quiconque". Ici encore, ces attitudes sont justifiées, aux yeux de Rémi et de ses collègues des services partenaires ${ }^{16}$, par le très jeune âge des enfants (considérés comme incapables de rester assis sans bouger et comme ayant besoin d'être rassurés par une attitude bienveillante), mais aussi par le souci de mettre à l'aise des parents de milieux populaires dont l'accès à ce type d'atelier n'est pas évident.

Ainsi, bien que le mode scolaire de socialisation ne soit pas absent de l'atelier, ce dernier prend une forme beaucoup moins scolaire que celle qui est habituellement à l'œuvre dans les conservatoires, et en particulier dans les cycles d'études musicales. Des recherches ont montré que la tendance générale supposée des conservatoires à la déscolarisation de leur enseignement (c'est-à-dire le souci de rendre l'enseignement de la musique moins "rigide", de valoriser sa dimension "sensible» plutôt que "technique», etc.) concernait davantage les discours et les objectifs des établissements et des enseignants que les pratiques pédagogiques effectives (Deslyper, 2013; Pégourdie \& Messina, 2013). Si l'atelier étudié ne reflète pas ce qui se fait le plus couramment au sein des conservatoires, on peut penser en revanche que sa présence dans un établissement de ce type est légitimée par cette évolution affichée. En effet, le directeur du conservatoire loue les qualités pédagogiques de Rémi (il souhaiterait même que les professeurs d'instrument de son établissement prennent exemple sur ses manières de faire) et il soutient pleinement l'atelier en affirmant que les enfants y pratiquent bien «de la musique». Au niveau de son établissement en général, il a œuvré à la diversification de l'offre, qui n'est plus seulement centrée sur la pratique instrumentale organisée en cycles mais qui intègre également plusieurs "ateliers " collectifs, dont certains sont accessibles sans "exigences techniques préalables» et ne sont pas évalués. II prend en outre ses distances avec les cursus classiques des conservatoires en disant souhaiter valoriser la "démarche » plutôt que le «résultat en soi », la pratique collective plutôt qu'individuelle, et le «corporel » et l'«oralité » plutôt que la musique «statique» et «intellectualisée».

Pour récapituler les caractéristiques de l'atelier étudié, on peut donc dire : 1) qu'il organise une activité musicale, mais sans que l'objectif musical soit prioritaire, sans que la musique soit la seule activité pratiquée, et sans qu'elle soit conçue comme une discipline à travailler; 2) qu'il est relativement éloigné de la forme scolaire de transmission des savoirs, même si les normes scolaires ne sont pas absentes en son sein. Nous allons montrer maintenant combien les parents qui fréquentent l'atelier se l'approprient différemment, en s'attachant davantage à certaines de ses caractéristiques plutôt qu'aux autres.

\section{DES APPROPRIATIONS PARENTALES SOCIALEMENT DIFFÉRENCIÉES}

Au cours des séances d'atelier, il y a peu d'interactions entre parents, entre enfants, et entre adultes et enfants n'entretenant pas de liens de parenté. On a plutôt affaire à une situation où chaque binôme parent-enfant fait face à Rémi et répond, ensemble, à ses propositions d'activités. Plus exactement, chaque parent joue un rôle d'intermédiaire entre Rémi et l'enfant, en guidant ce dernier dans la réalisation des activités. Tous les parents ne réagissent cependant pas de la même manière à l'atelier car ils se saisissent des aspects qui font davantage sens pour eux. Leurs interprétations de ce qui se passe lors 
des séances donnent ainsi un contenu particulier à la pratique musicale telle qu'elle est transmise à leurs enfants. Les parents de classes populaires ont davantage tendance à mettre au second plan les dimensions proprement musicales et scolaires de l'atelier alors que les parents de classes moyennes et supérieures insistent quant à eux davantage sur ces dimensions.

\section{Une dimension musicale plus ou moins repérée}

Dans les manières dont les parents de milieux populaires présentent l'atelier, la dimension musicale n'est jamais totalement absente. Mais ce qu'ils retiennent en priorité et ce qui motive principalement leur participation et leur goût pour l'activité ne relèvent pas à proprement parler de cette dimension. II s'agit en effet davantage à leurs yeux de trouver une occupation quelle qu'elle soit ainsi qu'un soutien éducatif face aux problèmes qu'ils rencontrent avec leurs enfants.

Les familles Macio (père maçon, mère sans profession et détentrice d'un CAP coiffure) et Milani (père magasinier, mère sans profession et détentrice d'un baccalauréat général17) permettent d'illustrer ces rapports à l'atelier musical. Mme Macio dit avoir accepté de participer à cet atelier parce qu'elle «cherchai[t] des solutions pour occuper [s]on fils en dehors de la maison » et elle rapproche alors cette activité de la fréquentation de «la garderie » où son fils se rend depuis peu. Mme Milani pense elle aussi que l'atelier permet de "voir autre chose que la maison" et d'occuper les enfants : “C'est vrai que de zéro à trois ans, on sait pas trop quoi faire pour égayer leur journée. Parce que c'est un peu toujours la même chose». Elle assimile pour sa part cette activité au lieu d'accueil enfants-parents de la ville qu'elle a un temps fréquenté : "Des ateliers en fait y en avait deux, y a celui-là et puis y a l'atelier "[nom du lieu d'accueil enfants-parents]" ". Toutes deux perçoivent en outre l'atelier musical moins comme un moyen d'accéder à une pratique musicale que comme un moyen de trouver une aide utile à l'éducation de leur enfant. Dans le cas de Mme Macio, il s'agit essentiellement de se saisir d'un outil de gestion des problèmes de son aîné qui souffre de «crises » de jalousie importantes suite à la naissance de sa petite sœur : «II fait des colères, vraiment des crises de colère où il s'arrête pas. C'est pas des pleurs, c'est des hurlements. [...] Je trouve que ça lui coupe un peu de ses idées qu'il arrive pas à se sortir de la tête». Dans le cas de Mme Milani, il s'agit de recueillir auprès de Rémi des avis et des conseils sur le développement de sa fille et sur sa manière de s'en occuper, comme elle le ferait auprès d'un pédiatre (mais comme les consultations de celui-ci sont «hyper rapides», elle estime qu'elles ne suffisent pas pour la «rassurer» et ne pas se «sentir seule» face à ses soucis éducatifs). Mme Milani s'approprie en outre l'atelier dans un sens particulièrement conforme aux objectifs du projet axés sur le lien parent-enfant, puisqu'elle estime qu'il lui a permis de se «rapprocher» de sa fille, de faire «les câlins, les bisous, autrement qu'à la maison». De plus, lorsqu'il lui arrive de reproduire chez elle des activités de l'atelier, il s'agit d'activités suscitant ce lien, qu'elle reproduit sans fond musical : «On faisait un truc là-bas, c'est monter sur les genoux et faire "tagada-tagada". Ça on a appris chez Rémi, et ça elle le demande tout le temps».

Si les familles de classes populaires appréhendent l'atelier dans ce sens, c'est parce qu'à la faiblesse - relative -

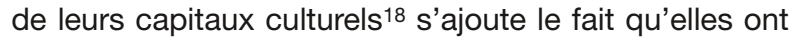
toutes été orientées par l'un des partenaires sociaux du projet. Dans les cas de Mme Macio (orientée par le Service petite enfance) et de Mme Milani (orientée par le Service social), ces modalités d'accès à l'atelier influencent d'autant plus leurs perceptions qu'elles ont été déterminantes dans leur fréquentation. Alors que pour les parents orientés de classes moyennes la suggestion du service partenaire joue uniquement un rôle d'information sur l'existence d'une activité qui les attire d'emblée parce qu'ils pensaient - mais plus tard - inscrire leurs enfants à un cours de musique, on a affaire dans le cas de Mmes Macio et Milani à un véritable accompagnement. L'une comme l'autre disent qu'elles n'auraient jamais pensé entrer dans un conservatoire sans cette orientation et toutes deux ont hésité à fréquenter l'atelier. C'est l'insistance des professionnelles des services partenaires qui les a convaincues. Ces deux mères se montrent en effet particulièrement réceptives à leurs discours, qui soulignent l'utilité de l'atelier pour occuper les enfants, gérer leurs émotions, faciliter le contact avec eux, etc. ${ }^{19}$ En particulier, Mme Milani (qui est suivie de longue date par la même assistante sociale) fait totalement confiance à ce discours, d'autant qu'elle se sent très peu assurée dans son rôle de mère : "Quand mon assistante sociale m'en a parlé, je me suis dit que si elle m'en parle, ça doit être bien»; «Moi j'y connais rien du tout, [...] qu'est-ce qu'on fait, qu'est-ce qu'on fait pas pour les enfants et tout ça. Euh je me laisse guider en gros ».

Du côté des familles de classes moyennes ${ }^{20}$ et supérieures, les appropriations de l'atelier sont davantage axées sur ses dimensions musicales. C'est le cas par exemple chez les familles Rugova (père et mère médecins), Torrez (père aide-soignant, mère infirmière) et Durand (mère ancienne danseuse et professeure de danse, reconvertie dans le «massage psycho-émotionnel », 
et détentrice d'un baccalauréat littéraire $\left.{ }^{21}\right)$. Les familles Rugova et Torrez ne font pas partie des familles orientées, ce qui, en plus de leurs caractéristiques sociales, diminue sans doute leurs chances d'appréhender l'atelier dans un sens extra-musical. Parmi les raisons de leur inscription à l'atelier, la dimension musicale apparaît prioritaire (même si non exclusive). Mme Rugova explique par exemple qu'elle et son mari souhaitaient que leur fille «participe dans des trucs de petite enfance [...], qu'elle soit avec d'autres enfants, surtout avec la musique», en continuité avec une pratique qu'elle avait déjà lorsqu'elle était enceinte (elle faisait écouter de la musique - «surtout la musique classique» - à son bébé). M. Torrez apprécie quant à lui l'atelier parce qu'il lui permet de consacrer «un temps donné » à la musique parmi les différentes activités qu'il accomplit avec ses enfants («Justement ce moment est bien parce qu'on fait que ça»). C'est aussi la dimension musicale qui est mise en avant lorsque ces parents décrivent le contenu de l'atelier : «On fait soit des mouvements, soit du rythme, soit il sort des instruments », dit par exemple M. Torrez. Contrairement aux enquêtés précédents, les parents de ces familles évoquent en outre le registre musical pratiqué lors de l'atelier. M. Torrez apprécie ainsi le fait que Rémi utilise des styles de musique variés et des chansons «pas connues » et, si Mme Torrez et Mme Rugova se sont senties mal à l'aise lors des premières séances auxquelles elles ont participé, c'est parce qu'elles ne connaissaient pas les chansons du fait de leurs origines étrangères (elles se sont ensuite initiées aux comptines françaises, Mme Rugova ayant même demandé à Rémi les titres des musiques utilisées pendant l'atelier pour les réécouter à la maison avec sa fille).

Contrairement à ces deux familles, la famille Durand a été orientée vers l'atelier (par le Service social fréquenté suite aux difficultés rencontrées par Mme Durand après la séparation avec son mari). De ce fait, Mme Durand souligne les fonctions de l'atelier, qui lui a permis de faire une parenthèse dans ses problèmes et de partager un moment agréable avec sa fille. Comme les mères de milieux populaires vues précédemment, elle adhère au discours institutionnel sur cette utilité : «[Mon assistante sociale] avait vu à cent pour cent juste quoi ». Mais, contrairement à elles, la proposition n'a fait dans son cas que confirmer une envie préexistante (“C'était évident pour moi que je mettrais Salomé à la musique à un moment ou à un autre»). De plus, les fonctions éducatives de l'atelier sont ici décrites par le prisme de ses caractéristiques musicales : "Je la vois encore faire quelques petits mouvements de rythme [...] et puis je lui prenais les mains, et puis on faisait ensemble»; «On est cent pour cent pour la musique et pour l'enfant, quoi».
Sa description de la salle où se tiennent les séances est en outre exclusivement centrée sur ses caractéristiques musicales: "La salle, là, avec les instruments de musique qui sont sur les étagères, le piano qui est au fond ». Enfin, comme les parents précédents, Mme Durand évoque le répertoire des musiques proposées par Rémi en soulignant le plaisir d'entendre des comptines déjà connues ou d'en apprendre de nouvelles.

\section{Une scolarisation plus ou moins forte de l'activité}

Si les parents de milieux populaires évoquent les apports éducatifs de l'atelier (ils apprécient par exemple que Rémi arrive à se faire obéir, à faire ranger les enfants, etc.), ils décrivent surtout ses dimensions informelles et récréatives. Pour Mme Macio, il s'agit avant tout d'«un bon moment à passer » dont elle apprécie le faible degré de directivité ("On est tous à même hauteur»; «[Rémi] il fait pas de discours, il attaque tout de suite»). Elle souligne à ce sujet le fait que l'atelier ne ressemble pas à ce qu'elle a pu observer dans les lieux habituels d'accueil de la petite enfance, caractérisés par une plus forte présence de consignes et de contraintes : "J'essayais d'imaginer ce que c'était, comme ils proposaient des fois en crèche ou en garderie [des activités musicales]. Ils expliquent aux enfants pendant un moment qu'est-ce qu'on va faire, qu'il faut quand même rester un petit peu à sa place pour pas déranger le cours. [...] Mais non du coup c'était pas comme ça du tout». Mme Milani apprécie elle aussi la «liberté» laissée aux enfants lors des séances et le fait qu'il n'y ait "pas trop de règles" : «À la maison on les formate un petit peu, les faire jouer de telle façon ou de telle façon, et là-bas c'est vrai que c'est vraiment libre, c'est eux qui décident vraiment [...]. Donc c'est ça qui est bien ". L'un de ses objectifs est que sa fille "s'amuse", "rigole", "se lâche», "se défoule» et fasse la «fofolle». Mme Macio et Mme Milani participent en outre à l'atelier sans beaucoup se soucier des possibilités pour leurs enfants de continuer une pratique musicale sur un plus long terme, alors qu'ils atteindront dès l'année suivante un âge qui ne leur permettra plus de fréquenter cet atelier. Enfin, lorsqu'elles parlent de Rémi, ces deux mères soulignent exclusivement des qualités favorisant le contact interpersonnel. Mme Macio le décrit ainsi comme "vraiment gentil et attentif à chaque personne, chaque maman, chaque enfant ", et Mme Milani souligne sa "bonne humeur", son aisance avec les enfants et son caractère «agréable».

Lors de nos observations, nous avons pu constater que les injonctions explicites ou implicites à respecter des 
règles étaient davantage le fait de certains parents que de Rémi, qui n'intervenait quasiment jamais, sauf pour seconder un parent déjà préoccupé par cet objectif (il lui est arrivé par exemple de proposer une activité à un enfant qu'un parent était en train de gronder, ce qui a eu pour effet de "calmer » l'enfant). L'analyse des entretiens menés avec les parents montre que ce sont ceux issus des milieux favorisés qui scolarisent ainsi l'activité, audelà des attentes de Rémi. Ces parents ont en particulier tendance à nuancer la liberté laissée aux enfants lors des séances et à interpréter les pratiques de Rémi comme un cadre implicite qui s'applique en douceur. Ainsi, par exemple, M. Torrez a bien constaté que sa fille «peut se lever et aller courir un peu » pendant l'activité («C'est pas trop trop gênant") mais ce constat - déjà nuancé par les termes employés - s'accompagne d'observations différentes : "Je sais pas comment dire, on est avec un professionnel avec notre enfant, il pose quand même des règles bien définies. [...] Des fois on a du mal à reprendre nos enfants parce que c'est nos enfants, mais là dès qu'ils vont dépasser un peu le cadre, Rémi arrive à les reprendre. [...] II intervient et ça se passe bien. Parce que l'enfant, hop, il réintègre tout de suite l'activité ou le cadre. Voilà, c'est pas grand-chose. D'où la pédagogie de Rémi qui est super forte". De même, pour Mme Durand, les règles sont loin d'être absentes lors des séances même si elles passent inaperçues : «Ça se fait tout seul». Elle parle ainsi de l'«autorité empathique» de Rémi qui lui permet de se faire obéir des enfants sans que son autorité prenne des formes trop visibles.

Les parents de ces familles scolarisent aussi l'atelier dans le sens où ils le perçoivent comme une première étape dans le cursus musical de leurs enfants (qui est d'ailleurs déjà un peu avancé pour deux d'entre eux, ce qui contribue aussi sans doute à cette lecture rétrospective de l'activité). De manière significative, M. Torrez considère ainsi que l'atelier fait partie du "cycle de l'éveil chez les tout-petits» et le désigne comme un «cours». Sa femme dit quant à elle avoir inscrit leurs filles à cette activité pour leur donner des «bases» en musique («Moi, dans ma logique à moi, je me disais que les bases de la musique autant les commencer le plus tôt possible»). Tous deux envisagent de les inciter à continuer la musique (ils se projettent en constatant que leurs filles ont des "facilités " dans ce domaine) et évoquent spontanément la perspective de les inscrire en "solfège" (c'est-à-dire dans la discipline la plus scolaire et écrite de l'apprentissage de la musique). Pour Mme Rugova et Mme Durand, dont les filles sont à présent inscrites en première année de premier cycle, l'atelier de Rémi participe aussi des bases musicales acquises par leurs filles. Mme Rugova estime même que sa fille - alors qu'elle n'a que sept ans "devrait être en deuxième année de solfège [...] parce qu'elle a fait l'éveil musical quand elle était bébé, donc elle avait pas besoin [de faire la première année]".

Enfin, si les parents de ces familles mentionnent, comme les parents précédents, la gentillesse de Rémi, ils ont une lecture moins personnelle de son rôle et insistent bien davantage sur le contenu de ce qu'il propose. Ainsi, pour Mme Rugova, ce n'est pas tant Rémi qui est «sympa» que l'activité qu'il anime : "[Lors de la première séance] on a vu qu'est-ce qu'il fait Rémi pendant ses cours et on a trouvé ça super sympa»; "Dès le petit âge, être en contact avec la musique, on trouvait ça super sympa». Quant à Mme Durand, elle se souvient moins de la personnalité de Rémi que de l'«univers » qu'il proposait (qui n'est d'ailleurs pas déconnecté dans son esprit d'une dimension scolaire, puisqu'elle fait référence ici à un tableau blanc sur lequel sont imprimées des portées servant aux cours de solfège destinés aux enfants plus grands) : «Rémi, je le vois très bien, $y$ avait le tableau derrière lui, il était assis là devant le tableau, avec ses instruments autour, avec ses marionnettes, avec tout son univers ".

\section{CONCLUSION}

L'atelier musical étudié se caractérise par une distance relative aux manières habituelles d'enseigner la musique au sein des conservatoires, dans la mesure où la musique et la forme scolaire n'y sont pas prioritaires. Les activités menées lors des séances sont en partie musicales, mais les objectifs particuliers qui encadrent l'atelier ainsi que la forme d'enseignement à l'œuvre tendent à faire passer au second plan voire à masquer cette dimension musicale. C'est ce que l'on observe dans les appropriations des parents de milieux populaires qui disposent moins que les autres des ressources permettant de coder l'activité dans un sens musical et qui, de surcroît, ont été orientés par des structures qui donnent justement un sens extra-musical à l'atelier. Quant à la forme scolaire, elle apparait peu présente au sein des séances. Les différences d'appropriations parentales sont donc moins liées ici aux capacités inégales des parents à repérer des implicites présents lors des séances qu'à leur tendance plus ou moins forte à scolariser une activité plus qu'elle ne l'est effectivement.

Là où d'autres articles de ce dossier ont analysé des décalages entre ce que l'enseignant pense faire et ce qu'il fait réellement, cet article a donc davantage mis en évi- 
dence des déplacements entre les caractéristiques d'une activité et ses appropriations. Les éventuels «malentendus" (Bautier \& Rayou, 2009; Bonnéry, 2007) observés sont ici tout autant le fait des parents de milieux populaires qui ne perçoivent pas (ou peu) la dimension musicale de l'activité, que des parents de milieux favorisés pour qui son éloignement relatif de la forme scolaire ne fait pas sens.

On peut faire l'hypothèse que ces appropriations différenciées renforcent, chez les enfants et dès le plus jeune âge, les inégalités d'accès à la musique (Octobre, 2004; Mennesson \& Julhe, 2012) et les inégalités de familiarisation à la forme scolaire (Joigneaux, 2009). En effet, bien que l'atelier mette l'apprentissage de la musique et les normes scolaires au second plan, certains parents se l'approprient dans un sens musical et scolaire, et sont ainsi en mesure de transmettre les attitudes et les perceptions correspondantes à leurs enfants. Si cet atelier n'est que l'une des pratiques socialisatrices des familles étudiées - et sans doute pas la plus déterminante compte tenu du temps somme toute limité qu'elles y consacrent -, il contribue donc, même modestement, à définir l'environnement dans lequel grandissent les enfants. C'est dans ce sens qu'il est en mesure de renforcer le processus de reproduction sociale qui est certainement en train de s'opérer au sein de ces familles.

\section{Géraldine Bois}

Geraldine.Bois@ens-lyon.fr Centre Max Weber, équipe DPCS, ENS de Lyon

\section{NOTES}

1 Les conservatoires sont les établissements publics d'enseignement spécialisé de la musique, de la danse et/ou du théâtre. Depuis 2006, ils sont classés en trois catégories : les "conservatoires à rayonnement communal ou intercommunal » (CRC), les «conservatoires à rayonnement départemental » $(\mathrm{CRD})$ et les "conservatoires à rayonnement régional » (CRR). Les premiers peuvent se contenter d'assurer l'enseignement d'une seule spécialité, alors que les autres doivent se charger d'au moins deux spécialités (CRD) ou de l'ensemble des trois spécialités (CRR).

2 L'expression "formation musicale » désigne ce qu'on appelle communément «solfège ».

3 Les cours d'instrument sont en partie individuels.

4 La plupart des enfants ont au moins un an, mais des bébés de quelques mois fréquentent aussi cet atelier. Un transat est d'ailleurs à disposition dans la salle où il se déroule.

5 Le terme est ici entre guillemets car, comme nous le verrons, l'atelier étudié ne prend pas la forme d'un enseignement au sens scolaire du terme. De même, le musicien intervenant qui l'anime est désigné comme un «enseignant » alors qu'il ne se définit pas comme tel. Ces termes sont malgré tout utilisés par commodité et parce qu'on a affaire à une situation formelle de transmission de savoirs dont il s'agit justement d'interroger la proximité à la forme scolaire. Les guillemets ne seront pas répétés dans la suite de l'article pour ne pas en alourdir la lecture.

6 Le thème de la «prévention précoce" est très présent dans les discours des professionnels de la petite enfance, comme en témoignent par exemple les nombreuses publications sur ce thème aux éditions Érès (dont plusieurs collections sont consacrées à l'enfance et à la parentalité).

7 La proposition d'inscription à l'atelier peut s'accompagner (ou, parfois, être remplacée par) d'autres propositions de soutien éducatif : participation à un autre atelier de parentalité, place de quelques matinées par semaine en halte-garderie, etc.

8 Document de présentation de l'atelier par les services de la ville.

9 Le directeur du conservatoire, qui n'intervient pas dans ce projet, dit ainsi de Rémi : "C'est lui qui est responsable de tout ça. Il est à la fois coordinateur et intervenant. II fait absolument tout le suivi d'organisation". L'intérêt de Rémi pour la petite enfance s'est construit au sein d'un parcours universitaire et professionnel particulier que nous ne détaillerons pas ici mais qui lui a permis de faire connaissance et de sympathiser avec la responsable petite enfance avec qui il a élaboré le projet.
10 Selon la nouvelle classification, il s'agit d'un CRC.

11 Tous les prénoms et les noms mentionnés dans cet article (enseignant et familles) ont été anonymés. L'enseignant n'est désigné que par un prénom car c'est comme cela qu'il est appelé par les parents, ce qui prend sens au regard de la relation pédagogique qui caractérise les séances et qui sera analysée plus loin. Rémi est âgé de quarante-neuf ans au moment de l'enquête. II est fonctionnaire territorial, assistant spécialisé d'enseignement artistique, employé à plein temps par le conservatoire.

12 II s'agit d'un lieu d'accueil enfants-parents, c'est-à-dire d'un lieu en accès libre où les enfants de moins de six ans, accompagnés d'un parent ou d'un adulte familier, viennent jouer, sous le regard de professionnels à l'écoute des parents qui le souhaitent.

13 Les jeux de caché-trouvé sont spécifiques à la petite enfance (ils sont un classique des activités de crèches et de petites sections d'école maternelle) et permettent, aux yeux des professionnels de ce secteur, d'expérimenter "la permanence de l'objet », les relations de causalité, ou la séparation nécessaire de l'enfant d'avec ses parents.

14 Pour la responsable du Centre information petite enfance et les assistantes sociales interrogées, cette poursuite n'est pas du tout un objectif.

15 Le musicien intervenant est en effet particulièrement enclin à critiquer l'enseignement traditionnel de la musique tel qu'il est pratiqué notamment dans les conservatoires (Lebon, 2014).

16 Celles-ci louent les capacités d'adaptation de Rémi au public de l'atelier et ses qualités relationnelles.

17 Si on a affaire ici à un niveau de diplôme plus élevé que dans la première famille, la famille Milani se caractérise aussi par une grande précarité économique (sa situation financière et résidentielle commence tout juste à se stabiliser au moment de l'entretien).

18 Ces familles ne sont pas dépourvues de ce type de capitaux, ce qui permet aussi d'expliquer le fait qu'elles fréquentent assidûment cet atelier-là. On observe notamment que tous les pères de ces familles pratiquent ou ont pratiqué un instrument de musique (à un "petit » niveau et, la plupart du temps, sans avoir suivi de cours).

19 En plus de l'atelier musical, le Service petite enfance et le Service social ont proposé à Mmes Macio et Milani un très grand nombre d'activités (le lieu d'accueil enfants-parents, la halte-garderie, la classe passerelle, un atelier massage pour bébés, etc.) et elles en ont acceptées certaines. Les autres familles orientées, dont l'accompagnement vers l'atelier a été moins serré, ne mentionnent pas 
une telle multiplicité d'incitations. C'est sans doute parce qu'elles font moins l'objet d'une surveillance institutionnelle, leurs normes éducatives étant socialement plus proches de celles véhiculées par les professionnels des services sociaux (Garcia, 2011).

20 Les familles de classes moyennes de notre population se caractérisent notamment par un capital culturel important : activités de loisirs culturels fréquentes et diversifiées, et présence chez au moins I'un des deux parents d'une pratique musicale ou artistique poussée dans un cadre institutionnel. La famille Torrez est considérée comme appartenant aux classes moyennes car, contrairement aux familles de classes populaires de notre population, l'un des deux parents (ici la mère) a une activité qui relève des «professions intermédiaires". De plus, M. Torrez exerce son métier d'aide-soignant à temps partiel. Le reste du temps, il s'occupe de ses enfants mais il a aussi une activité de musicien, que l'on peut qualifier de semiprofessionnelle (il joue dans un groupe qui fait quelques concerts et il a enregistré un CD).

21 Les propriétés sociales du père ne sont pas connues car son évocation avec Mme Durand lors de l'entretien était rendue difficile par les circonstances de la séparation du couple quelques années auparavant (situation de violence conjugale).

\section{BIBLIOGRAPHIE}

BAUTIER É. \& RAYOU P. (2009). Les inégalités d'apprentissage. Programmes, pratiques et malentendus scolaires. Paris: PUF.

BERGER P. \& LUCKMANN T. (1986). La construction sociale de la réalité. Paris : Méridiens.

BOIS G., GARDON S., GIRAUD F. \& RAYNAUD A. (2014). La démocratisation de l'accès aux pratiques artistiques à travers l'apprentissage dans les établissements d'enseignement artistique. Rapport de recherche remis à la Région Rhône-Alpes.

BONNÉRY S. (2007). Comprendre l'échec scolaire. Élèves en difficultés et dispositifs pédagogiques. Paris : La Dispute.

CHAMBOREDON J.-C. \& Prévôt J. (1973). «Le "métier d'enfant". Définition sociale de la prime enfance et fonctions différentielles de l'école maternelle". Revue française de sociologie, no14, p.295-335.

DESLYPER R. (2013). Les élèves guitaristes des écoles de "musiques actuelles» : analyse du passage de l'autodidaxie à l'enseignement pédagogique. Thèse de doctorat, sociologie, université Lumière-Lyon 2.

GARCIA S. (2011). Mères sous influence. De la cause des femmes à la cause des enfants. Paris : La Découverte.

GARNIER P. (2006). "L'enfant et le sport : classements d'âge et pratiques sportives". In R. Sirota, Éléments pour une sociologie de l'enfance. Rennes: Presses universitaires de Rennes, p.205-214.
GARNIER P. (2009). "Préscolarisation ou scolarisation? L'évolution institutionnelle et curriculaire de l'école maternelle". Revue française de pédagogie, no169, p.5-15.

JOIGNEAUX C. (2009). «La construction de l'inégalité scolaire dès l'école maternelle". Revue française de pédagogie, no169, p. 17-28.

LEBON F. (2014). Profession «musicien intervenant» à l'école. Paris : L'Harmattan.

LUC J.-N. (1997). L'invention du jeune enfant au xxe siècle. De la salle d'asile à l'école maternelle. Paris : Belin.

MENNESSON C. \& Julhe S. (2012). «L'art (tout) contre le sport? La socialisation culturelle des enfants des milieux favorisés ». Politix, n॰99, p.109-128.

MINISTÈRE DE LA CULTURE ET DE LA COMMUNICATION (2008). Schéma national d'orientation pédagogique de l'enseignement initial de la musique. Paris : Direction de la musique, de la danse, du théâtre et des spectacles.

OCTOBRE S. (2004). Les loisirs culturels des 6-14 ans. Paris : La Documentation française.

PÉGOURDIE A. \& MESSINA L. (2013). «L'art à l'épreuve de la pédagogie. Ethnographie de deux classes de formation musicale». In M. Perrenoud, Travailler, produire, créer. Entre l'art et le métier. Paris : L'Harmattan, p.75-92.

VINCENT G. (dir.) (1994). L'éducation prisonnière de la forme scolaire? Lyon : Presses universitaires de Lyon. 